\title{
A Class of Loss Functions of Catenary Form
}

\author{
D. E. Raeside ${ }^{1,2}$ and R. J. Owen ${ }^{1,3}$
}

Received June 18, 1971; revised June 12, 1972

\begin{abstract}
The catenary form of loss function is considered in the framework of Bayesian decision theory. The mathematical tractability of this form seems to be unrecognized; it contains quadratic loss as a limiting case. For various probability distributions expressions are given for posterior analysis, and limiting properties are investigated.
\end{abstract}

KEY WORDS: Parameter estimation; Bayesian estimation theory; loss functions; non-mean-square error criterion; quadratic loss function.

\section{INTRODUCTION}

In the Bayesian approach to statistics (1-4) linear and quadratic loss functions have been widely discussed and their engineering applications well treated (5-8). In addition, a limited literature characterizing broad classes of loss functions has also appeared ${ }^{(9-11)}$. The purpose of this note is to give results on a specific one-parameter family of loss functions.

In this note a quantity thought of as a random variable will be denoted by a capital letter, the lower case form of the letter being reserved for a realization or fixed value of that random variable.

Let $W$ denote the unknown (scalar) parameter of interest and $F(\cdot)$ the

${ }^{1}$ The University of Michigan, Ann Arbor, Michigan.

${ }^{2}$ Present address: Department of Radiological Sciences, University of Oklahoma Health Sciences Center, Oklahoma City, Oklahoma.

${ }^{3}$ Present address: Department of Statistics, University College of Wales, Penglais, Aberystwyth, Wales, U. K. 
cumulative distribution function for $W$ (this may be a prior or a posterior distribution, depending on the context). It is assumed that the moment generating function of $W$,

$$
M(t) \equiv \int_{-\infty<w<\infty} e^{w t} d F(w)
$$

exists in some interval containing 0 , say $-\alpha<t<\beta(\alpha, \beta>0)$. Leibniz's rule for differentiation under the integral sign (extended to Stieltjes integrals) applies in the same interval (Ref. 12, p. 240); hence, for $r=0,1, \ldots$ the $r^{\text {th }}$ derivative of $M(t), M^{(r)}(t)$, exists and is given by

$$
M^{(r)}(t)=\int_{-\infty<w<\infty} w^{r} e^{w t} d F(w) \quad(-\alpha<t<\beta)
$$

In particular, for $r=0,1, \ldots$ the $r^{\text {th }}$ moment of $W, \mu_{r}{ }^{\prime}$, exists and equals $M^{(r)}(0)$. The $r^{\text {th }}(r=0,1, \ldots)$ central moment of $W, E_{W}\left\{\left(W-\mu_{1}{ }^{\prime}\right)^{r}\right\}$, will be denoted by $\mu_{r}$. The alternative notation $\mu$ and $\sigma^{2}$ will be used for $\mu_{1}^{\prime}$ and $\mu_{2}$, respectively.

It will be more convenient to work with the moment generating function of $W-\mu$ :

$$
N(t)=e^{-\mu t} M(t)
$$

Of course the derivatives of $N(t)$, like those of $M(t)$, exist for $-\alpha<t<\beta$.

The loss function to be considered is

$$
L(w, d)=[\cosh a(w-d)]-1
$$

where $a$ is a specified positive constant. Notice that the function of $x$, $(\cosh a x)-1$, is nonnegative (vanishing only at $x=0$ ), strictly increasing in $|x|$, symmetric about $x=0$, and strictly convex.

In the usual Bayesian decision-theoretic framework let $\rho$ denote the Bayes risk,

$$
\rho \equiv \inf _{d} E_{W}\{L(W, d)\}
$$

and $\hat{d}$ a value of $d$ (if it exists) which attains this infimum; $\hat{d}$ is called a Bayes estimator of $w$.

\section{POSTERIOR ANALYSIS}

Taking the usual differentiation approach for minimization and using (2) for $r=1,2$, it follows that for $a<\min (\alpha, \beta)$ the Bayes estimator $d$ for the loss function (4) is unique and

$$
\hat{d}=\mu+(1 / 2 a) \ln [N(a) / N(-a)], \quad \rho=[N(a) N(-a)]^{1 / 2}-1
$$


In the case where the distribution of $W$ is symmetric about the mean, $N(a)=N(-a)$ and (6) simplifies to

$$
\hat{d}=\mu, \quad \rho=N(a)-1
$$

The first of these results, simply that $\hat{d}=\mu$, is in agreement with the result in Ref. 9 for a convex, symmetric loss function and a distribution symmetric about its mean.

These expressions are now evaluated for various distributions of $W$ which are commonly utilized as prior distributions. In what follows $f(\cdot)$ denotes a probability density function and $p(\cdot)$ a probability mass function.

\subsection{Normal}

$$
\begin{aligned}
f(w)= & (h / 2 \pi)^{1 / 2} \exp \left[-\frac{1}{2} h(w-\mu)^{2}\right], \\
& \quad-\infty<w<\infty \quad(-\infty<\mu<\infty, \quad h>0) \\
\hat{d}= & \mu, \quad \rho=\exp \left(\frac{1}{2} a^{2} h^{-1}\right)-1
\end{aligned}
$$

\subsection{Uniform}

$$
\begin{aligned}
f(w) & =\frac{1}{2} \theta^{-1}, \quad \mu-\theta<w<\mu+\theta \quad(-\infty<\mu<\infty, \quad \theta>0) \\
\hat{d} & =\mu, \quad \rho=(1 / a \theta)(\sinh a \theta)-1
\end{aligned}
$$

\subsection{Gamma}

$$
f(w)=\left[\lambda^{r} / \Gamma(r)\right] w^{r-1} e^{-\lambda w}, \quad w>0 \quad(\lambda>0, \quad r>0)
$$

Then for $a<\lambda$

$$
\hat{d}=(r / 2 a) \ln [(\lambda+a) /(\lambda-a)], \quad \rho=\lambda^{r}\left(\lambda^{2}-a^{2}\right)^{-r / 2}-1
$$

\subsection{Beta (First Kind)}

$$
\begin{aligned}
f(w) & =[1 / B(p, q)] w^{p-1}(1-w)^{q-1}, \quad 0<w<1 \quad(p>0, \quad q>0) \\
\hat{d} & =(1 / 2 a) \ln [\Phi(p, p+q ; a) / \Phi(p, p+q ;-a)] \\
\rho & =[\Phi(p, p+q ; a) \Phi(p, p+q ;-a)]^{1 / 2}-1
\end{aligned}
$$

Here we use the notation $\Phi(\cdot, \cdot ; \cdot)$ to designate the degenerate hypergeometric function (Ref. 13, p. 1058). 


\subsection{Binomial}

$$
\begin{aligned}
p(w)= & \left(\begin{array}{l}
n \\
w
\end{array}\right) p^{w} q^{n-w}, \quad w=0,1, \ldots, n \\
& (0<p<1, \quad q \equiv 1-p, \quad n=1,2, \ldots) \\
\hat{d}= & (n / 2 a) \ln \left[\left(p e^{a}+q\right) /\left(p e^{-a}+q\right)\right] \\
\rho= & {\left[1+4 p q \sinh ^{2}(a / 2)\right]^{n / 2}-1 }
\end{aligned}
$$

\subsection{Negative Binomial}

$$
\begin{array}{r}
p(w)=\left(\begin{array}{c}
r+w-1 \\
w
\end{array}\right) p^{r} q^{w}, \quad w=0,1, \ldots \\
(0<p<1, \quad q \equiv 1-p, \quad r=1,2, \ldots)
\end{array}
$$

Then for $a<\ln (1 / q)$

$$
\begin{aligned}
& \hat{d}=(r / 2 a) \ln \left[\left(1-q e^{-a}\right) /\left(1-q e^{a}\right)\right] \\
& \rho=\left[p^{2} /\left(1-q e^{-a}\right)\left(1-q e^{a}\right)\right]^{r / 2}-1
\end{aligned}
$$

\subsection{Poisson}

$$
\begin{aligned}
p(w) & =\lambda^{w} e^{-\lambda} / w !, \quad w=0,1, \ldots \quad(\lambda>0) \\
\hat{d} & =(\lambda / a) \sinh a, \quad \rho=\exp [\lambda(\cosh a-1)]-1
\end{aligned}
$$

\section{ASYMPTOTIC PROPERTIES}

Here it is shown that asymptotically as $a \rightarrow 0$, the loss function (4) "behaves like" the quadratic loss function

$$
L_{1}(w, d)=\frac{1}{2} a^{2}(w-d)^{2}
$$

In what follows the symbols $\sim$ and $O$ will have their usual meaning. Denote the Bayes estimator and Bayes risk associated with $L_{1}$ by $\hat{d}_{1}$ and $\rho_{1}$, respectively. It is well known that

$$
\hat{d}_{1}=\mu \quad \text { and } \quad \rho_{\mathbf{1}}=\frac{1}{2} a^{2} \sigma^{2}
$$

Referring to (6), it follows by l'Hôspital's rule [the conditions for which are satisfied because of (2)] that

$$
\hat{d} \rightarrow \hat{d}_{1} \quad \text { as } \quad a \rightarrow 0
$$


Referring to (A.9), (A.10), and (A.12), it may be asserted by Taylor's theorem that for any $\gamma_{1}$ between 0 and $\gamma$ and any $a$ such that $0 \leqslant|a| \leqslant \gamma_{1}$ there is some $\theta$ between 0 and $a$ such that

$$
\rho(a)=\frac{1}{2} a^{2} \mu_{2}+(1 / 4 !) a^{4} \rho^{(4)}(\theta) \quad\left(0 \leqslant|a| \leqslant \gamma_{1}<\gamma\right)
$$

Here $\theta$ is a function of $a$ and of the datum $x$. Regarding $\rho^{(4)}(\theta)$ as a function of $a$ for given $x$, it follows from (A.12) and (A.10) that $\rho^{(4)}(\theta)$ is bounded on $0 \leqslant|a| \leqslant \gamma_{1}$ and tends to $\mu_{4}$ as $a \rightarrow 0$. Therefore

$$
\rho=\frac{1}{2} a^{2} \mu_{2}+O\left(a^{4}\right) \quad \text { and } \quad\left(\rho-\rho_{1}\right) /\left[\frac{1}{2}\left(\rho+\rho_{1}\right)\right] \sim \frac{1}{12} a^{2} \mu_{4} / \mu_{2}
$$

It is of interest to note that stemming from the inequality $1+\frac{1}{2} x^{2} \leqslant \cosh$ $x \leqslant 1+\frac{1}{2} x^{2}+(1 / 24) x^{3} \sinh x$ (which is true for all $x$ ),

$$
0 \leqslant \frac{\rho-\rho_{1}}{\frac{1}{2}\left(\rho+\rho_{1}\right)} \leqslant \frac{a}{12 \mu_{2}} \int_{-\infty<w<\infty}(w-\mu)^{3} \sinh a(w-\mu) d F(w) \equiv \epsilon(a)
$$

$\epsilon(a)$ may be expressed in terms of $N(\cdot)$ :

$$
\epsilon(a)=\left(a / 24 \mu_{2}\right)\left[N^{(3)}(a)-N^{(3)}(-a)\right]
$$

This expression may be used to calculate expressions for $\epsilon(a)$, for example, when $W$ has a normal distribution with mean $\mu$ and variance $\sigma^{2} \equiv 1 / h$,

$$
\epsilon(a)=\frac{1}{12} a^{2}\left(3+a^{2} \sigma^{2}\right) \sigma^{2} \exp \left(\frac{1}{2} a^{2} \sigma^{2}\right)
$$

Differentiation of (14) establishes that $\epsilon(a)$ is an increasing function of $a$ and an expansion of $\epsilon(a)$ by Taylor's theorem establishes that

$$
\epsilon(a) \sim \frac{1}{2} a^{2} \mu_{4} / \mu_{2} \quad \text { as } \quad a \rightarrow 0
$$

Notice that this is the same as the asymptotic form of $\left(\rho-\rho_{1}\right) /\left[\frac{1}{2}\left(\rho+\rho_{1}\right)\right]$ and so, denoting this relative difference by $\eta$, it is natural to be curious about the asymptotic behavior of $(\epsilon-\eta) /\left[\frac{1}{2}(\epsilon+\eta)\right]$. A Taylor expansion approach establishes that as $a \rightarrow 0$

$$
(\epsilon-\eta) /\left[\frac{1}{2}(\epsilon+\eta)\right] \sim(1 / 15) a^{2} \mu_{4}^{-1}\left(2 \mu_{6}+5 \mu_{3}^{2}\right)
$$

\section{APPENDIX. THE DERIVATIVES OF $\rho$}

This topic is of interest for a Taylor expansion of $\rho$ and it is presented here to avoid a digression in the body of the article. The definitions and results up to and including Eq. (7) are assumed. Let

$$
y \equiv y(a) \equiv N(a) N(-a) \quad \text { so } \quad \rho \equiv \rho(a)=y^{1 / 2}-1
$$


Because of (2) all derivatives of $y$ exist and are continuous for $|a|<\gamma$ where $\gamma=\min (\alpha, \beta)$. For a function $f$ let $(\partial / \partial a)^{r} f$ be denoted by $f^{(r)}$; then it follows from Leibniz's rule for differentiating a product that for $n=0,1, \ldots$

$$
y^{(n)}(a)=\sum_{r=0}^{n}\left(\begin{array}{l}
n \\
r
\end{array}\right)(-1)^{r} N^{(r)}(-a) N^{(n-r)}(a) \quad(|a|<\gamma)
$$

Pairing off terms from the ends of the sum (A.2), it follows that

$$
\begin{aligned}
& y^{(n)}(0)=0 \\
& y^{(n)}(0)=2 \sum_{r=0}^{(n / 2)-1}(-1)^{r}\left(\begin{array}{l}
n \\
r
\end{array}\right) \mu_{r} \mu_{n-r}+\left(\begin{array}{c}
n \\
\frac{1}{2} n
\end{array}\right)(-1)^{n / 2} \mu_{n / 2}^{2} \quad\left(\begin{array}{ll}
n & \text { even })
\end{array}\right.
\end{aligned}
$$

The derivatives of $y$ will now be used to calculate the derivatives of $\rho$. The approach is to write (A.1) in the form

$$
y=(1+\rho)^{2}
$$

from which is obtained, by Leibniz's rule, denoting $\partial / \partial a$ by $D$,

$$
y^{(n)}=\sum_{r=0}^{n}\left(\begin{array}{l}
n \\
r
\end{array}\right) D^{r}(1+\rho) D^{n-r}(1+\rho) \quad(|a|<\gamma)
$$

Pairing off terms from the ends, this reduces to (for $|a|<\gamma$ )

$$
\begin{aligned}
& y^{(n)}=2 \sum_{r=0}^{(n-1) / 2}\left(\begin{array}{l}
n \\
r
\end{array}\right)(1+\rho)^{(r)}(1+\rho)^{(n-r)} \quad(n \quad \text { odd }) \\
& y^{(n)}=2 \sum_{r=0}^{(n / 2)-1}\left(\begin{array}{l}
n \\
r
\end{array}\right)(1+\rho)^{(r)}(1+\rho)^{(n-r)}+\left(\begin{array}{c}
n \\
\frac{1}{2} n
\end{array}\right)\left[(1+\rho)^{n / 2}\right]^{2} \quad(n \quad \text { even })
\end{aligned}
$$

Using (A.3) and (A.7), it follows by induction that

$$
\rho^{(n)}(0)=0 \quad(n \quad \text { odd })
$$

Using (A.4), (A.8), and (A.9), the even derivatives are calculated sequentially and it is found that

$$
\begin{gathered}
\rho^{(2)}(0)=\mu_{2}, \quad \rho^{(4)}(0)=\mu_{4}, \quad \rho^{(6)}(0)=\mu_{6}-10 \mu_{3}^{2}, \\
\rho^{(8)}(0)=280 \mu_{2} \mu_{3}^{2}-56 \mu_{3} \mu_{5}+\mu_{8}
\end{gathered}
$$


When the distribution of $W$ is symmetric about the mean, all odd central moments are zero and referring to (7) expanded as a Taylor series, there follows the simple solution for the even central moments

$$
\rho^{(2 r)}(0)=\mu_{2 r} \quad(r=1,2, \ldots)
$$

This result may also be arrived at by induction from (A.4) and (A.8).

Equating the expressions for $y^{(n)}(a)$ in (A.2) and (A.6), it follows by an induction argument on $n$ that

$$
\rho^{(n)}(a) \text { exists and is continuous, } \quad[n=0,1, \ldots ; \quad(0 \leqslant|a|<\gamma)] \text { (A.12) }
$$

\section{REFERENCES}

1. M. H. DeGroot, Optimal Statistical Decisions, McGraw-Hill, New York (1970).

2. I. H. LaValle, An Introduction to Probability, Decision and Inference, Holt, Rinehart and Winston, New York (1970).

3. D. V. Lindley, Introduction to Probability and Statistics from a Bayesian Viewpoint; Part 1, Probability; Part 2, Inference, Cambridge University Press, London (1965).

4. H. Raiffa and R. Schlaifer, Applied Statistical Decision Theory, Division of Research, Graduate School of Business Administration, Harvard University, Boston (1961).

5. C. W. Helstrom, Statistical Theory of Signal Detection, Pergamon Press, Oxford (1968).

6. H. L. Van Trees, Detection, Estimation and Modulation Theory, Wiley, New York (1968).

7. A. J. Viterbi, Principles of Coherent Communication, McGraw-Hill, New York (1966).

8. R. Deutsch, Estimation Theory, Prentice-Hall, Englewood Cliffs, N. J. (1965).

9. M. H. De Groot and M. M. Rao, "Bayes Estimation with Convex Loss," Ann. Math. Stat. 34:839-846 (1963).

10. S. Sherman, "Non-Mean-Square Error Criteria," IRE Trans. Information Theory IT-4:125-126 (1958).

11. M. Zahai, General Error Criteria, IEEE Trans. Information Theory IT-10:94-95 (1964).

12. D. V. Widder, The LaPlace Transform, Princeton University Press, Princeton N.J. (1946).

13. I. S. Gradshteyn and I. M. Ryzhik, Table of Integrals, Series, and Products, Academic Press, New York (1965). 